\title{
Ruthenium-Catalyzed Synthesis of 3-Substituted Quinolines from 2-Aminobenzyl Alcohol and Aldehydes
}

\author{
Chan Sik Cho, ${ }^{\dagger, *}$ Wen Xiu Ren, and Sang Chul Shim* \\ ${ }^{\dagger}$ Research Institute of Industrial Technology, Kyungpook National University, Daegu 702-701, Korea. "E-mail: cscho@knu.ac.kr \\ Department of Applied Chemistry, College of Engineering, Kyungpook National University, Daegu 702-701, Korea \\ *E-mail:scshim@knu.ac.kr \\ Received September 28, 2005
}

Key Words : Aldehydes, 2-Aminobenzyl alcohol, Oxidative cyclization, Ruthenium catalyst, 3-Substituted quinolines

It is known that many quinoline containing compounds exhibit a broad spectrum of pharmacological and biological activities. ${ }^{1}$ During the course of our studies on transition metal-catalyzed N-heterocyclization, we have also reported on synthesis of quinolines via ruthenium-catalyzed alkyl or alkanol group transfer from alkylamines or alkanolamines to $\mathrm{N}$-atom of anilines (amine exchange reaction) ${ }^{2}$ and palladium-catalyzed coupling and cyclization between 2iodoaniline and propargylic alcohols. ${ }^{3}$ Furthermore, in connection with this report, it has recently been found that carbonyl compounds (or secondary alcohols) are coupled with primary alcohols in the presence of a ruthenium catalyst and $\mathrm{KOH}^{4-6}$ These newly developed coupling reactions could also be applied to modified Friedläender quinoline synthesis via ruthenium- and palladium-catalyzed coupling and cyclization of 2-aminobenzyl alcohol with ketones and secondary alcohols. ${ }^{7-9}$ Under these circumstances, the present work was disclosed during the course of the extension of this protocol to the reaction of 2-aminobenzyl alcohol with aldehydes. Herein, we describe a ruthenium-catalyzed oxidative coupling and cyclization between 2-aminobenzyl alcohol and aldehydes leading to 3substituted quinolines.

Initial attempts for the oxidative cyclization of 2-aminobenzyl alcohol (1) with octyl aldehyde (2a) were examined under several conditions. Treatment of 1.5 equivalent of 1 with $2 \mathbf{a}$ in dioxane in the presence of a catalytic amount of $\mathrm{RuCl}_{2}\left(\mathrm{PPh}_{3}\right)_{3}$ and $\mathrm{KOH}$ at $80{ }^{\circ} \mathrm{C}$ for $20 \mathrm{~h}$ afforded 3hexylquinoline (3a) in only $22 \%$ yield with concomitant

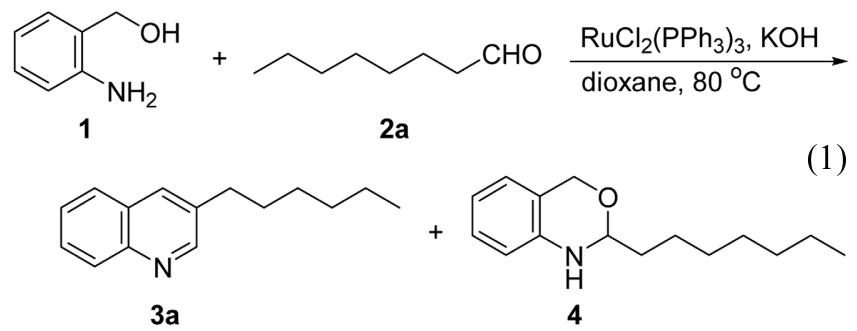

formation of 2-heptyl-1,4-dihydro-2H-benzo[d][1,3]oxazine (4) $(2 \%)$ (Eq. 1). ${ }^{10}$ However, step-by-step procedure, an initial treatment of $\mathbf{1}$ in the presence of $\mathrm{RuCl}_{2}\left(\mathrm{PPh}_{3}\right)_{3}$ and $\mathrm{KOH}$ in dioxane for $15 \mathrm{~h}$ and subsequent addition of $\mathbf{2 a}$ to the mixture followed by stirring for $5 \mathrm{~h}$ at the same temperature resulted in an increased yield of $\mathbf{3 a}(58 \%)$, whereas 4 remained constant. In contrast to our recent report on ruthenium-catalyzed synthesis of quinolines from $\mathbf{1}$ and secondary alcohols, the addition of 1-decene as a sacrificial hydrogen acceptor did not affect the yield of $\mathbf{3 a}(58 \%)$.

Next, various aldehydes were subjected to cyclize with $\mathbf{1}$ in order to investigate the reaction scope and several representative results are summarized in Table 1. With straight aldehydes (2a-2c) the 3-substituted quinolines (3a-3c) were formed in the range of $58-66 \%$ yields with the minimal formation of oxazines on GLC analysis. The product yield was not significantly affected by the chain length of 2a-2c. The reaction proceeds likewise with hydrocinnamaldehyde (2d) having phenyl group at position 3 to give the corresponding quinoline $\mathbf{3 d}$ in similar yield. In the reaction of isovaleraldehyde (2e) and 3-phenylbutyraldehyde (2f) which have substituents such as methyl and phenyl at position 3, the corresponding 3-isopropylquinoline (3e) and 3-(1phenylethyl)quinoline (3f) were also obtained in $59 \%$ and $67 \%$ yields, respectively. Lower reaction rate and yield were observed with 3,3-dimethylbutyraldehyde (2g), which has two substituent at position 3 . The reaction of phenylacetaldehyde (2h), which has a phenyl substituent at position 2, with 1 also proceeds to give 3-phenylquinoline (3h) and the quinoline yield was lower than when straight and $\beta$ substituted aldehydes were used. Finally, we attempted the oxidative cyclization of $\mathbf{1}$ with primary alcohol instead of aldehyde for the wide availability of substrates. ${ }^{4}$ However, similar treatment of $\mathbf{1}$ with 3-methyl-2-butanol instead of $\mathbf{2 e}$ under the employed conditions gave $3 \mathbf{e}$ in only $5 \%$ yield without any identifiable products.

In summary, we have demonstrated that 2-aminobenzyl alcohol can be oxidatively cyclized with an array of 
Table 1. Ruthenium-catalyzed synthesis of quinolines $\mathbf{3}$ from $\mathbf{1}$ and $\mathbf{2}^{a}$

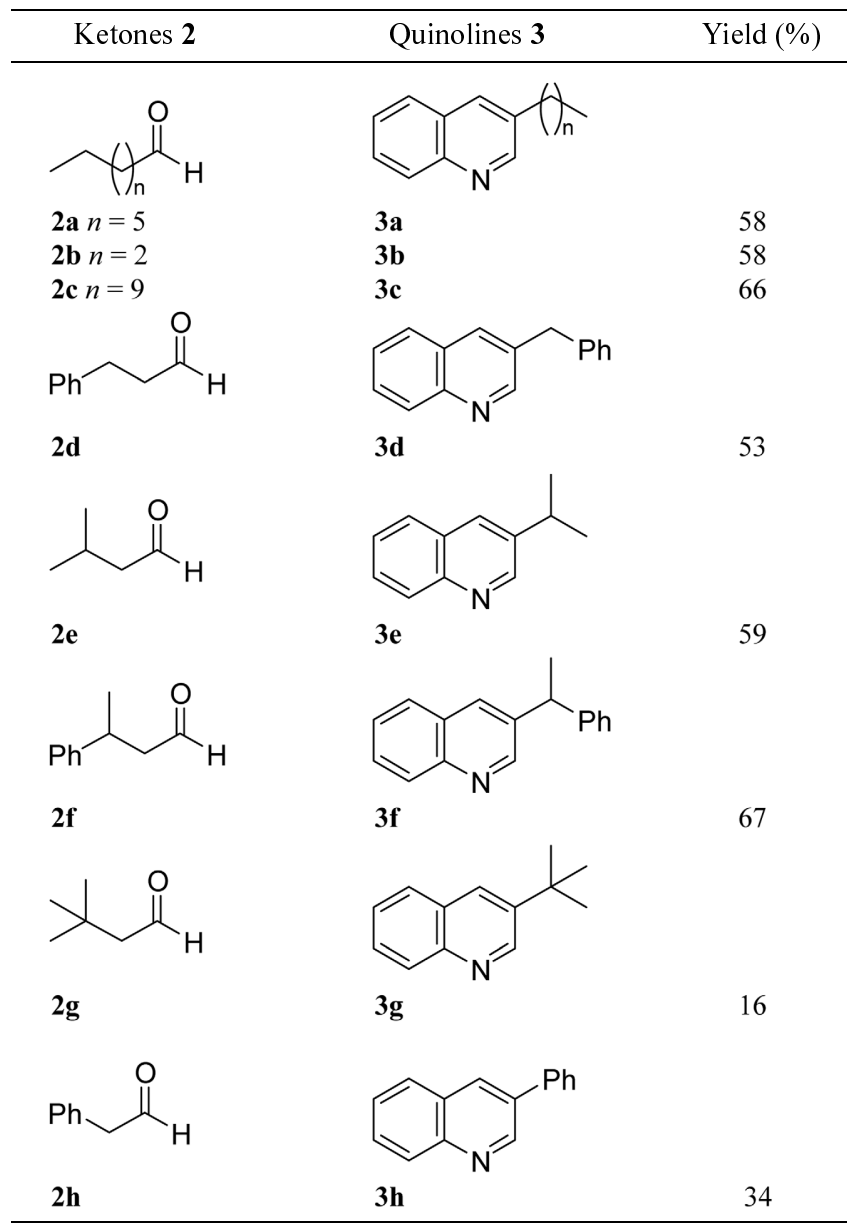

${ }^{a}$ Reaction conditions: $1(1.5 \mathrm{mmol}), \mathrm{RuCl}_{2}\left(\mathrm{PPh}_{3}\right)_{3}(0.03 \mathrm{mmol}), \mathrm{KOH}(2$ $\mathrm{mmol})$, dioxane $(5 \mathrm{~mL}), 80^{\circ} \mathrm{C}$, for $15 \mathrm{~h} ; 2(1 \mathrm{mmol})$, dioxane $(3 \mathrm{~mL}), 80$ ${ }^{\circ} \mathrm{C}$, for $5 \mathrm{~h}$.

aldehydes in the presence of a ruthenium catalyst and $\mathrm{KOH}$ to give 3-substituted quinolines in good yields.

\section{Experimental Section}

${ }^{1} \mathrm{H}$ and ${ }^{13} \mathrm{C}$ NMR (400 and $100 \mathrm{MHz}$ ) spectra were recorded on a Bruker Avance Digital 400 spectrometer using TMS as an internal standard. GLC analyses were carried out with a Shimadzu GC-17A instrument equipped with a CBP10S25-050 column (Shimadzu, fused silica capillary column, $0.33 \mathrm{~mm} \times 25 \mathrm{~m}, 0.25 \mu \mathrm{m}$ film thickness) using nitrogen as carrier gas. The isolation of pure products was carried out via thin layer chromatography (silica gel $60 \mathrm{GF}_{254}$, Merck). Commercially available organic and inorganic compounds were used without further purification.

General experimental procedure. A mixture of 2aminobenzyl alcohol $(0.185 \mathrm{~g}, 1.5 \mathrm{mmol}), \mathrm{RuCl}_{2}\left(\mathrm{PPh}_{3}\right)_{3}$ $(0.029 \mathrm{~g}, 0.03 \mathrm{mmol})$ and $\mathrm{KOH}(0.112 \mathrm{~g}, 2 \mathrm{mmol})$ in dioxane $(5 \mathrm{~mL})$ was placed in an organic reactor (Radleys Discovery Technologies) and allowed to react at $80{ }^{\circ} \mathrm{C}$ for 15 $\mathrm{h}$. To the mixture was added aldehyde $(1 \mathrm{mmol})$ in dioxane $(3 \mathrm{~mL})$. The mixture was stirred at the same temperature for
$5 \mathrm{~h}$ and filtered through a short silica gel column (ethyl acetate-hexane mixture) to eliminate inorganic salts. Removal of the solvent left a crude mixture, which was separated by thin layer chromatography (silica gel, ethyl acetate-hexane mixture) to give quinolines 3. All products prepared by the above procedure were characterized spectroscopically as shown below.

3-Hexylquinoline (3a). Oil; ${ }^{1} \mathrm{H} \mathrm{NMR}\left(\mathrm{CDCl}_{3}\right) \delta 0.89$ (t, $J$ $=6.6 \mathrm{~Hz}, 3 \mathrm{H}), 1.29-1.34(\mathrm{~m}, 4 \mathrm{H}), 1.35-1.40(\mathrm{~m}, 2 \mathrm{H}), 1.68-$ $1.75(\mathrm{~m}, 2 \mathrm{H}), 2.79(\mathrm{t}, J=7.8 \mathrm{~Hz}, 2 \mathrm{H}), 7.49-7.53(\mathrm{~m}, 1 \mathrm{H})$, $7.63-7.67(\mathrm{~m}, 1 \mathrm{H}), 7.76(\mathrm{~d}, J=8.0 \mathrm{~Hz}, 1 \mathrm{H}), 7.90(\mathrm{~d}, J=1.0$ $\mathrm{Hz}, 1 \mathrm{H}), 8.08(\mathrm{~d}, J=8.5 \mathrm{~Hz}, 1 \mathrm{H}), 8.78(\mathrm{~d}, J=2.0 \mathrm{~Hz}, 1 \mathrm{H})$; ${ }^{13} \mathrm{C} \mathrm{NMR}\left(\mathrm{CDCl}_{3}\right) \delta 14.04,22.54,28.83,31.07,31.61$, $33.17,126.46,127.25,128.16,128.44,129.10,134.05$, 135.35, 146.71, 152.10 .

3-Propylquinoline (3b). Oil; ${ }^{1} \mathrm{H} \mathrm{NMR}\left(\mathrm{CDCl}_{3}\right) \delta 0.87(\mathrm{t}$, $J=7.3 \mathrm{~Hz}, 3 \mathrm{H}), 1.57-1.66$ (m, 2H), 2.63 (t, $J=7.8 \mathrm{~Hz}, 2 \mathrm{H})$, $7.38(\mathrm{t}, J=7.5 \mathrm{~Hz}, 1 \mathrm{H}), 7.53(\mathrm{t}, J=7.5 \mathrm{~Hz}, 1 \mathrm{H}), 7.63(\mathrm{~d}, J=$ $8.0 \mathrm{~Hz}, 1 \mathrm{H}), 7.77(\mathrm{~s}, 1 \mathrm{H}), 7.99$ (d, $J=8.5 \mathrm{~Hz}, 1 \mathrm{H}), 8.66$ (d, $J$ $=2.0 \mathrm{~Hz}, 1 \mathrm{H}) ;{ }^{13} \mathrm{C} \mathrm{NMR}\left(\mathrm{CDCl}_{3}\right) \delta 14.04,24.59,35.55$, $126.91,127.67,128.54,128.93,129.33,134.70,135.46$, $146.95,152.33$.

3-Decylquinoline (3c). Oil; ${ }^{1} \mathrm{H}$ NMR $\left(\mathrm{CDCl}_{3}\right) \delta 0.79(\mathrm{t}, J$ $=6.8 \mathrm{~Hz}, 3 \mathrm{H}), 1.18-1.33(\mathrm{~m}, 14 \mathrm{H}), 1.59-1.67(\mathrm{~m}, 2 \mathrm{H}), 2.70$ $(\mathrm{t}, J=7.8 \mathrm{~Hz}, 2 \mathrm{H}), 7.41-7.45(\mathrm{~m}, 1 \mathrm{H}), 7.55-7.59(\mathrm{~m}, 1 \mathrm{H})$, 7.67-7.69 (m, 1H), $7.84(\mathrm{~d}, J=1.5 \mathrm{~Hz}, 1 \mathrm{H}), 8.02(\mathrm{~d}, J=8.6$ $\mathrm{Hz}, 1 \mathrm{H}), 8.70(\mathrm{~d}, J=2.0 \mathrm{~Hz}, 1 \mathrm{H}) ;{ }^{13} \mathrm{C} \mathrm{NMR}\left(\mathrm{CDCl}_{3}\right) \delta$ $14.49,23.06,29.57,29.69,29.81,29.94,29.97,31.49$, $32.26,33.58,127.00,127.68,128.61,129.04,129.25$, $134.81,135.83,146.76,152.19$.

3-Benzylquinoline (3d). Viscous oil; ${ }^{1} \mathrm{H} \mathrm{NMR}\left(\mathrm{CDCl}_{3}\right) \delta$ $3.98(\mathrm{~s}, 2 \mathrm{H}), 7.06-7.11(\mathrm{~m}, 3 \mathrm{H}), 7.15-7.19(\mathrm{~m}, 2 \mathrm{H}), 7.33-$ $7.36(\mathrm{~m}, 1 \mathrm{H}), 7.49-7.52(\mathrm{~m}, 1 \mathrm{H}), 7.56(\mathrm{~d}, J=8.0 \mathrm{~Hz}, 1 \mathrm{H})$, 7.71 (s, $1 \mathrm{H}), 7.98$ (d, $J=8.5 \mathrm{~Hz}, 1 \mathrm{H}), 8.68$ (d, $J=2.0 \mathrm{~Hz}$, $1 \mathrm{H}) ;{ }^{13} \mathrm{C} \mathrm{NMR}\left(\mathrm{CDCl}_{3}\right) \delta 39.63,127.01,127.15,127.89$, $128.54,129.18,129.35,129.37,129.46,134.29,135.39$, 140.06, 147.14, 152.38 .

3-Isopropylquinoline (3e). Oil; ${ }^{1} \mathrm{H} \mathrm{NMR}\left(\mathrm{CDCl}_{3}\right) \delta 1.28$ $(\mathrm{d}, J=7.0 \mathrm{~Hz}, 6 \mathrm{H}), 2.98-3.09(\mathrm{~m}, 1 \mathrm{H}), 7.40-7.45(\mathrm{~m}, 1 \mathrm{H})$, $7.54-7.58(\mathrm{~m}, 1 \mathrm{H}), 7.69(\mathrm{~d}, J=8.0 \mathrm{~Hz}, 1 \mathrm{H}), 7.85(\mathrm{~d}, J=2.3$ $\mathrm{Hz}, 1 \mathrm{H}), 8.01$ (d, $J=8.0 \mathrm{~Hz}, 1 \mathrm{H}), 8.75$ (d, $J=2.3 \mathrm{~Hz}, 1 \mathrm{H})$; ${ }^{13} \mathrm{C} \mathrm{NMR}\left(\mathrm{CDCl}_{3}\right) \delta 24.01,32.23,126.98,127.86,128.63$, 129.06, 129.24, 132.48, 141.53, 146.97, 151.32.

3-(1-Phenylethyl)quinoline (3f). Viscous oil; ${ }^{1} \mathrm{H}$ NMR $\left(\mathrm{CDCl}_{3}\right) \delta 1.70(\mathrm{~d}, J=7.0 \mathrm{~Hz}, 3 \mathrm{H}), 4.29(\mathrm{q}, J=7.0 \mathrm{~Hz}, 1 \mathrm{H})$, 7.15-7.22 (m, 3H), 7.25-7.28 (m, 2H), 7.42-7.46 (m, 1H), 7.57-7.62 (m, 1H), 7.69 (d, $J=8.0 \mathrm{~Hz}, 1 \mathrm{H}), 7.89(\mathrm{~d}, J=2.0$ $\mathrm{Hz}, 1 \mathrm{H}), 8.08$ (d, $J=8.5 \mathrm{~Hz}, 1 \mathrm{H}), 8.79$ (d, $J=2.0 \mathrm{~Hz}, 1 \mathrm{H})$; ${ }^{13} \mathrm{C}$ NMR $\left(\mathrm{CDCl}_{3}\right) \delta 22.04,42.97,126.97,127.07,128.05$, $128.10,128.49,129.10,129.26,129.55,133.43,139.33$, $145.34,147.26,152.24$.

3-tert-Butylquinoline (3g). Oil; ${ }^{1} \mathrm{H} \mathrm{NMR}\left(\mathrm{CDCl}_{3}\right) \delta 1.46$ (s, 9H), 7.50-7.55 (m, 1H), 7.64-7.68 (m, 1H), $7.79(\mathrm{~d}, J=$ $8.0 \mathrm{~Hz}, 1 \mathrm{H}), 8.04(\mathrm{~d}, J=2.5 \mathrm{~Hz}, 1 \mathrm{H}), 8.08$ (d, $J=8.5 \mathrm{~Hz}$, $1 \mathrm{H}), 9.03(\mathrm{~d}, J=2.5 \mathrm{~Hz}, 1 \mathrm{H}) ;{ }^{13} \mathrm{C} \mathrm{NMR}\left(\mathrm{CDCl}_{3}\right) \delta 31.33$, $34.21,126.90,128.08,128.26,129.05,129.24,131.23$, $143.71,146.66,150.44$. 
3-Phenylquinoline (3h). Viscous oil; ${ }^{1} \mathrm{H} \mathrm{NMR}\left(\mathrm{CDCl}_{3}\right) \delta$ $7.30(\mathrm{t}, J=7.3 \mathrm{~Hz}, 3 \mathrm{H}), 7.37-7.45(\mathrm{~m}, 3 \mathrm{H}), 7.56-7.60(\mathrm{~m}$, $3 \mathrm{H}), 7.33(\mathrm{~d}, J=8.0 \mathrm{~Hz}, 1 \mathrm{H}), 8.04(\mathrm{~d}, J=8.0 \mathrm{~Hz}, 1 \mathrm{H}), 8.15$ $(\mathrm{d}, J=2.0 \mathrm{~Hz}, 1 \mathrm{H}), 9.06(\mathrm{~d}, J=2.0 \mathrm{~Hz}, 1 \mathrm{H}) ;{ }^{13} \mathrm{C} \mathrm{NMR}$ $\left(\mathrm{CDCl}_{3}\right) \delta 127.47,127.80,128.42,128.55,129.44,129.59$ $(\times 2), 129.90,133.80,134.22,138.14,147.49,150.12$.

Acknowledgment. The present work was supported by BK-21 in 2003 and a Research Foundation Grant (KRF2002-070-C00055). C.S.C. gratefully acknowledges a Research Professor Grant of Kyungpook National University (2004).

\section{References}

1. (a) Jones, G. Comprehensive Heterocyclic Chemistry; Katritzky, A. R., Rees, C. W., Eds.; Pergamon: New York, 1984; Vol. 2, p 395. (b) Arcadi, A.; Chiarini, M.; Giuseppe, S. D.; Marinelli, F. Synlett 2003, 203, and references cited therein. (c) Cho, S. Y.; Ahn, J. H.; Ha, J. D.; Kang, S. K.; Baek, J. Y.; Han, S. S.; Shin, E. Y.; Kim, S. S.; Kim, K. R.; Cheon, H. G.; Choi, J.-K. Bull. Korean Chem. Soc. 2003, 24, 1455.

2. (a) Cho, C. S.; Oh, B. H.; Shim, S. C. Tetrahedron Lett. 1999, 40, 1499. (b) Cho, C. S.; Oh, B. H.; Shim, S. C. J. Heterocycl. Chem. 1999, 36, 1175. (c) Cho, C. S.; Kim, J. S.; Oh, B. H.; Kim, T.-J.; Shim, S. C. Tetrahedron 2000, 56, 7747. (d) Cho, C. S.; Oh, B. H.; Kim, J. S.; Kim, T.-J.; Shim, S. C. Chem. Commun. 2000, 1885. (e) Cho, C. S.; Kim, T. K.; Kim, B. T.; Kim, T.-J.; Shim, S. C. J. Organomet. Chem. 2002, 650, 65.
3. Cho, C. S. J. Organomet. Chem. 2005, 690, 4094.

4. (a) Cho, C. S.; Kim, B. T.; Kim, T.-J.; Shim, S. C. J. Org. Chem. 2001, 66, 9020. (b) Cho, C. S.; Kim, B. T.; Kim, T.-J.; Shim, S. C. Tetrahedron Lett. 2002, 43, 7987. (c) Cho, C. S.; Kim, B. T.; Kim, H.-S.; Kim, T.-J.; Shim, S. C. Organometallics 2003, 22, 3608 .

5. For palladium-catalyzed version of coupling between ketones and primary alcohols leading to a-alkylated ketones: Cho, C. S. J. Mol. Cat. A: Chem. 2005, 240, 55.

6. Taguchi, K.; Nakagawa, H.; Hirabayashi, T.; Sakaguchi, S.; Ishii, Y. J. Am. Chem. Soc. 2004, 126, 72.

7. (a) Friedläender, P. Chem. Ber. 1882, 15, 2572. (b) For a review, see: Cheng, C.-C.; Yan, S.-J. Org. Reactions 1982, 28, 37. (c) Muchowski, J. M.; Maddox, M. L. Can. J. Chem. 2004, 82, 461.

8. (a) Cho, C. S.; Kim, B. T.; Kim, T.-J.; Shim, S. C. Chem. Commun. 2001, 2576. (b) Cho, C. S.; Kim, B. T.; Choi, H.-J.; Kim, T.-J.; Shim, S. C. Tetrahedron 2003, 59, 7997. (c) Cho, C. S.; Ren, W. X.; Shim, S. C. Bull. Korean Chem. Soc. 2005, 26 , 1286.

9. (a) Motokura, K.; Mizugaki, T.; Ebitani, K.; Kaneda, K. Tetrahedron Lett. 2004, 45, 6029. (b) Martínez, R.; Brand, G. J.; Ramón, D. J.; Yus, M. Tetrahedron Lett. 2005, 46, 3683. (c) Taguchi, K.; Sakaguchi, S.; Ishii, Y. Tetrahedron Lett. 2005, 46, 4539.

10. We separately synthesized $\mathbf{4}$ for identification by simple stirring 1 and 2a in dioxane at $80{ }^{\circ} \mathrm{C}$ for $4 \mathrm{~h}$. Oil; ${ }^{1} \mathrm{HNMR}\left(\mathrm{CDCl}_{3}\right) \delta 0.89(\mathrm{t}$, $J=6.8 \mathrm{~Hz}, 3 \mathrm{H}), 1.29-1.34(\mathrm{~m}, 8 \mathrm{H}), 1.43-1.57(\mathrm{~m}, 2 \mathrm{H}), 1.63-1.79$ $(\mathrm{m}, 2 \mathrm{H}), 4.53(\mathrm{t}, J=5.5 \mathrm{~Hz}, 1 \mathrm{H}), 4.80(\mathrm{~d}, J=14.6 \mathrm{~Hz}, 1 \mathrm{H}), 4.94$ (d, $J=14.6 \mathrm{~Hz}, 1 \mathrm{H}), 6.66(\mathrm{~d}, J=8.0 \mathrm{~Hz}, 1 \mathrm{H}), 6.77-6.81(\mathrm{~m}, 1 \mathrm{H})$ $6.91(\mathrm{~d}, J=7.5 \mathrm{~Hz}, 1 \mathrm{H}), 7.06(\mathrm{t}, J=7.5 \mathrm{~Hz}, 1 \mathrm{H}) ;{ }^{13} \mathrm{C} \mathrm{NMR}$ $\left(\mathrm{CDCl}_{3}\right) \delta 14.48,23.04,24.91,29.58,29.87,32.16,35.60,68.06$, $84.79,117.60,120.02,123.00,125.38,127.71,141.98$. 